\title{
Evaluación de tres índices bióticos en un río subtropical de montaña (Tucumán - Argentina)
}

\author{
H. R. Fernández $z^{1,4}$, F. Romero ${ }^{2}$, M. B. Vece ${ }^{1,3}$, V. Manzo', C. Nieto ${ }^{1,4}$, M. Orce ${ }^{1}$ \\ ${ }^{2}$ Facultad de Ciencias Naturales e Instituto Miguel Lillo. U.N.T Miguel Lillo 205- 4000 S. M. de Tucuinán- \\ Tucumán- Argentina. e-mail hugof@selway.umt.ar. \\ ${ }^{2}$ Fundación Miguel Lillo. Miguel Lillo 25 1- 4000 S.M.de Tucuinán- Tucumán- Argentina. \\ ${ }^{3}$ Centro de Investigaciones y Transferencia en Química Aplicada. (C.I.Q.). Facultad de C.N. e Inst. Miguel Lillo. \\ UNT. Miguel Lillo 205- 4000 S.M.de Tucumán- Tucumán- Argentina. e-mail maritas@csnat.unt.edu.ar \\ ${ }^{4}$ CONICET
}

\section{RESUMEN}

Se presenta en este trabajo la relación entre variables ambientales e índices bióticos de un río que no evidencia entradas puntuales de contaminantes, aunque hay explotación forestal y extensas áreas con cultivos en el resto de la cuenca. Este río puede convertirse en referencia para una extensa área subandina oriental que constituye una ecorregión conocida como Yungas correspondiente a la selva subtropical de montaña caracterizada por importantes precipitaciones estacionales (>1.000 min). Se tomaron muestras del zoobentos y se midieron variables tísico-químicas cada dos meses durante un año en cuatro estaciones ubicadas en el tramo medio del río estudiado, (Lules -Tucumán - Argentina).

Se calcularon tres índices bióticos, BMWP', ASP'I" y EPT (modificando los dos primeros para la región que nos ocupa), que se relacionaron con las variables fisico-químicas del agua. Esto permitió establecer correlaciones entre índices bióticos, variables fisico-químicas e índices entre sí. Se usó un análisis de correspondencia canónica y se determinó que las variables más importantes en este tramo del río a lo largo del año son la materia orgánica y los sólidos totales.

Palabras clave: río subtropical, Yungas, BMWP', ASPT', EPT, zoobentos

\begin{abstract}
Environmental variables and biotic indica were applied to $u$ Subtropical Mountain stream in Tucumán (Argentina). The river exhibits no obvious point entry ofpollutaiits, although lumber extraction and extensively cultivated areas are found throughout the basin. Its ecological status makes it an appropriate reference point for a large oriental sub-Andeun area. the Yungas eco-region. This ecoregion corresponds to the subtropical mountain forest. characterized by abundant seasonal precipitation $(>1000 \mathrm{~mm})$. Benthic samples were taken and physical variables were measured every two months over a period of one year at four sites in middle reaches of the studied river (Lules - Tucumán-Argentina). Three biotic indices were calculated, $B M W P$ ', ASPT' and EPT. The first two were adapted to the region considered. Indices were related to water physical and chemical variables. Correlations between hiotic indices and physical and chemicul variables and aniong the biotic indices themselves were then established, using a Canonicul Correspondence Analysis (CCA). Organic matter and total suspended solids were the most important variables in this stretch of the river throughout the year.
\end{abstract}

Keywords: subtropical river, Yungas, BMWP : ASPT', EPT, zoobenthos

\section{INTRODUCCIÓN}

Un volumen importante del agua de los ríos en el mundo se origina por escurrimiento a través de áreas cultivadas. Este tiene una influencia desproporcionadamente alta sobre la calidad de las aguas si lo comparamos con el que proviene de la escorrentía de cuencas con cobertura vegetal natural 
(Dodds, 1997).Sin embargo, esto no se refleja en el interés de los investigadores que han enfatizado, en general, sus estudios ecológicos en ríos bajo influencia de bosques naturales templados (Covich, 1988, Dodds, 1997). Actualmente esta tendencia parece revertirse, debido a la aparición de numerosos trabajos con énfasis en ríos de áreas impactadas en regiones subtropicales y tropicales (Domínguez y Fernandez, 1998, Caicedo y Palacio, 1998, Jacobsen, 1998, Salinas et al., 1999).

En este sentido y debido a la importancia creciente de los problemas de contaminación, es necesario determinar qué parámetros abióticos y/o bióticos pueden utilizarse para evaluar el estado de los cuerpos de agua. Entre los métodos de evaluación, los biológicos permiten un

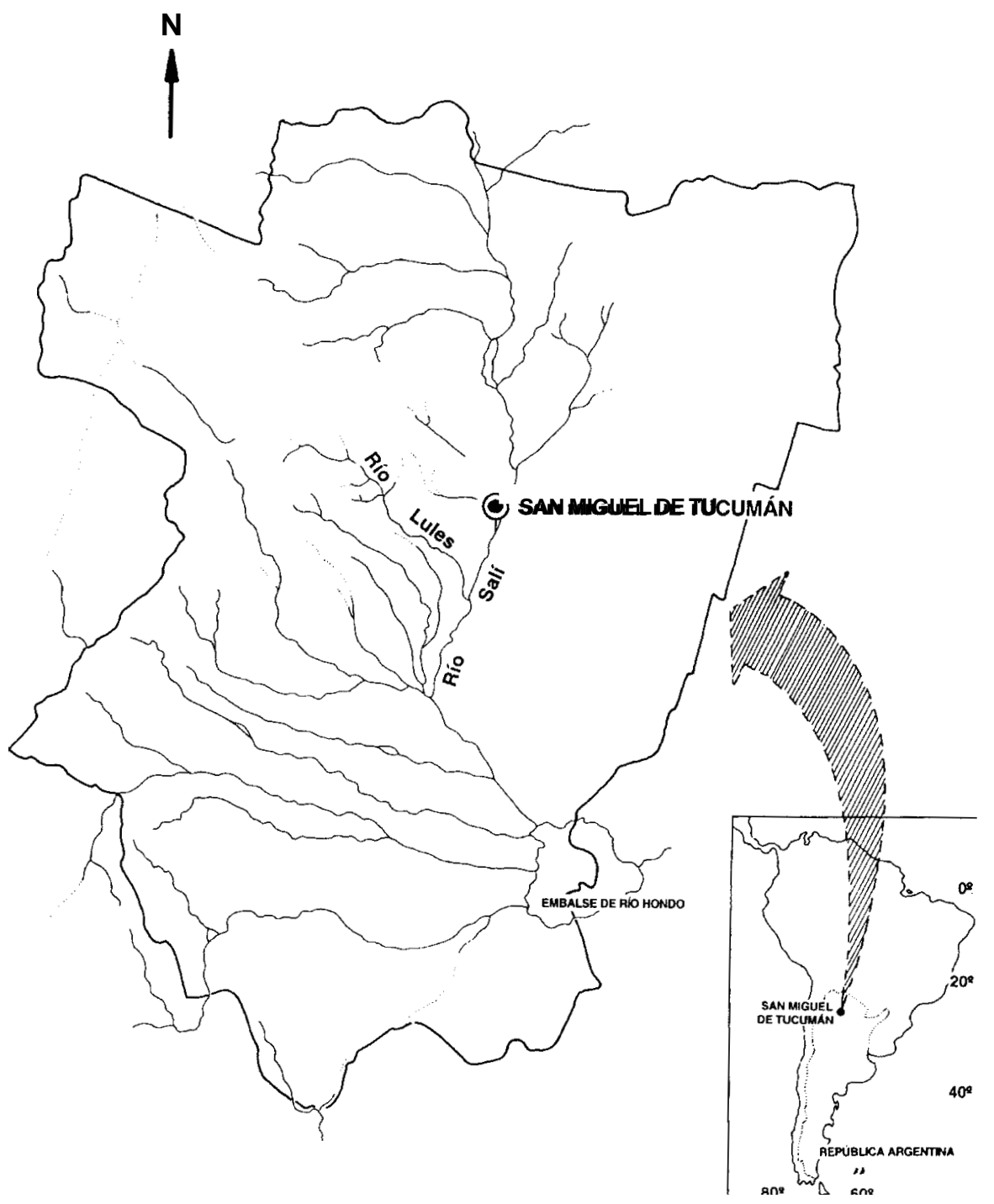

Figura 1. Ubicación del río Lules en la provincia de Tucumán y su localización en América del Sur. Location of the River Lules in the pro vince of Tucumán and in Southamerica (inset). 
diagnóstico rápido de la situación en un curso de agua, utilizando bioindicadores e índices bióticos (Alba-Tercedor y Sánchez-Ortega, 1988). Estos utilizan, principalmente a los invertebrados acuáticos (Cairns \& Pratt, 1993) o peces (Karr et al., 1985) entre otros y son ampliamente usados en Europa y en menor grado en Estados Unidos.

Para adoptar un índice, desarrollado originalmente para otra región, es necesario realizar estudios de validación, así como adaptar los puntajes de los taxones indicadores (Graça y Coimbra, 1998). Uno de estos índices empíricos, el Biological Monitoring Working Party o BMWP' (Alba-Tercedor y Sánchez-Ortega, 1988) fue adaptado en una primera etapa a la provincia de Tucumán (Argentina) utilizando los macroinvertebrados presentes en la región, para poder dar respuestas rápidas a las demandas de nuestro entorno económico - social (Domínguez y Fernández, 1998).

En el presente trabajo se probaron algunos de estos índices, en el río Lules (Fig. 1) perteneciente a la cuenca del río Salí, donde hay sospechas de contaminación difusa por el desarrollo de actividades agrícola-ganaderas en la zona ribereña adyacente, además de un progresivo deterioro de la cuenca por erosión atribuible a la tala indiscriminada (Moyano y Movia, 1988).

Se presentan los resultados de un año de trabajo de un proyecto multidisciplinario que intenta conocer la dinámica de la comunidad y los facto- res físico - químicos que actúan sobre un tramo de río, incluyendo el área marginal. En este último aspecto se pretende desarrollar una medida de su calidad (Braioni et al. 1994) atendiendo a la marcada influencia que tiene la misma sobre el ecosistema Iótico.

\section{ÁREA DE ESTUDIO}

La provincia de Tucumán ocupa una posición central dentro del noroeste argentino y se encuentra surcada por numerosos ríos, la mayoría de los cuales nacen en el oeste montañoso. Sobre las laderas y en la base de estas montañas se extiende la ecorregión de las Yungas (Morales et al., 1995) caracterizándose por incluir un rango altitudinal entre 400 y 3000 (msnm), con precipitaciones de origen orográfico superiores a los 1000 $\mathrm{mm}$ anuales, máximos de $3000 \mathrm{~mm}$ en algunos sitios y un carácter marcadamente estacional, (durante los meses de verano se concentra $80 \%$ de las mismas). Por esta razón esta ecorregión es considerada el área principal de producción de agua para las zonas urbanizadas de la región de llanura de la provincia.

Para llevar a cabo este trabajo se seleccionó el río Lules, con un caudal promedio anual de 6.07 $\mathrm{m}^{3} / \mathrm{s}$ (Evarsa, 1997) que al igual que numerosos ríos de la provincia, drena en la cuenca endorreica

Tabla 1. Características físicas y biológicas de las estaciones de muestreo. Physical and biological characteristics of sampling stations

\begin{tabular}{|c|c|c|c|c|}
\hline & RSJ & RLH & RLJ & $\mathbf{R L}$ \\
\hline Altitud (msnm) & 890 & 860 & 670 & 470 \\
\hline Profundidad promedio (m) & 0.16 & 0.23 & 0.31 & 0.22 \\
\hline Ancho promedio $(\mathrm{m})$ & 4.12 & 10.35 & 14.44 & 27.27 \\
\hline Temperatura promedio del agua (“C) & 18.80 & 19.35 & 21.41 & 22.63 \\
\hline $\begin{array}{l}\text { Tipo de Sustrato en el canal } \\
\text { (Klemm } \text { et al.,1990) }\end{array}$ & grava & Bloques y grava & Bloques y grava & Bloques y grava \\
\hline Cobertura algal (Cladophora sp.) & $\begin{array}{l}\text { Alta (invierno } \\
\text { y primavera) }\end{array}$ & escasa & $\begin{array}{l}\text { escasa } \\
\text { (primavera) }\end{array}$ & Media \\
\hline $\begin{array}{l}\text { Formación vegetal circundante } \\
\text { (Morales et al., 1995) }\end{array}$ & Selva montana & Selva montana & Selva montana & Selva pedemontana \\
\hline Tipo y grado de alteración ribereña & $\begin{array}{l}\text { actividades agrícolas } \\
\text { ganaderas (Alta) }\end{array}$ & $\begin{array}{l}\text { actividades } \\
\text { recreacionales } \\
\text { (Baja) }\end{array}$ & $\begin{array}{l}\text { actividades } \\
\text { recreacionales } \\
\text { (Baja) }\end{array}$ & $\begin{array}{l}\text { actividades } \\
\text { recreacionales } \\
\text { (Baja) }\end{array}$ \\
\hline
\end{tabular}


Salí - Dulce (Fig. 1). La subcuenca del río Lules, con un área de $4192 \mathrm{~km}^{2}$, tiene una importante superficie (38\%) alterada por explotación forestal y cultivos que se asocia principalmente a las áreas fluviales vecinas (Moyano y Movia, 1988). Este río atraviesa dos estratos de vegetación (Tabla 1) de las Yungas que presentan especies arbóreas de gran porte (Morales et al., 1995).

Se seleccionaron cuatro estaciones de inuestreo (Fig. 2), ubicadas a una distancia promedio aproximada de 10 km entre sí, identificándolas como: Río San Javier (RSJ), Río La Hoyada (RLH) ambas ubicadas sobre afluentes que confluyen, junto con el Río Potrero de las Tablas, para formar el Río Las Juntas (RLJ) donde ubicamos la tercera estación y finalmente luego de la confluencia de pequeños arroyos de primer orden, cambia su nombre a Río Lules (RL), donde se ubicó la cuarta estación. Las características de cada una de ellas se describen en la tabla 1 y corresponden al tramo medio del río (orden 4 y 5).

\section{MATERIAL Y MÉTODOS}

Se realizaron seis muestreos bimestrales desde mayo de 1998 hasta abril de 1999 y en cada uno de ellos se tomaron muestras de zoobentos y agua. Para la colección de macroinvertebrados se utilizó una red en forma de D con malla de 300 $\mu \mathrm{m}$ (Domínguez y Fernández, 1998). En cada estación se removió el sustrato delante de la red. La misma se colocó en distintos puntos formando una transecta oblicua al eje del río hasta el medio del cauce. Esta operación se realizó dos veces en cada estación, utilizándose como unidad de esfuerzo estandarizada un tiempo de 10 minutos para cada transecta. El material colectado fue fijado con formol al $4 \%$ en el campo. En el laboratorio se realizó la determinación taxonómica del material según el requerimiento de los índices a utilizar: hasta el nivel de familia en la mayoría de los casos (Insecta), que permitieron calcular los índices BMWP' y ASPT'. Mientras que en Ephemeroptera, Plecoptera y Trichoptera la determinación se realizó hasta nivel específico para obtener el índice EPT.
Se midieron, in situ, $\mathrm{pH}$ y temperatura utilizando un peachímetro (Methrom 704) y conductividad utilizando un conductímetro (Methrom E587). La alcalinidad se determinó mediante titulación volumétrica. Se midió además velocidad de corriente por método de flotación, ancho y profundidad con regla y ruleta, calculando el caudal a partir de estas mediciones (Hynes, 1970).

A cada una de las muestras de agua, en el laboratorio y siguiendo los métodos propuestos por APHA (1 992), se le hicieron las siguientes determinaciones: sodio y potasio (Método fotométrico de emisión de llama), calcio y magnesio (Método titulométrico de EDTA), cloruro (Método argentométrico), fosfato (Método del ácido ascórbico) y sólidos totales (Secado a $103-105^{\circ} \mathrm{C}$ ). Se utilizó la metodología propuesta por Rodier (1 989) para la determinación de sulfato (Método nefelométrico), oxígeno disuelto (Método de Winkler modificación de Alsterberg), materia orgánica (Oxígeno consumido por la materia orgánica) y nitrato (Método del salicilato sódico).

Se calcularon los siguientes índices bióticos: BMWP' (Biological Monitoring Working Party) modificado para la región por Domínguez y Fernández (1998) su variante el ASPT' (Average Score Per Taxon) que surge de dividir el BMWP' por el número de taxones involucrados en el cálculo (Walley \& Hawkes, 1997), y por primera vez en la región se utilizó el índice EPT calculado sobre el número de especies presentes de Ephemeroptera, Plecoptera y Trichoptera (Klemm et al., 1990).

Con los valores obtenidos para el BMWP' se estableció también la clase de calidad del agua (Tabla 3) basándose en las cinco establecidas por este índice (Domínguez y Fernández, 1998).

Con las matrices de datos integrados de todo el año de: presencia y ausencia de los taxones en cada estación y de las variables medidas, se aplicó el Análisis de Correspondencia Canónica (ACC) utilizando el programa CANOCO (Ter Braak, 1986, 1988). Las variables fueron transformadas utilizando $\log (\mathrm{x}+1)$ mientras que la matriz de los taxones se utilizó sin transformación.

Posteriormente para establecer la relación existente entre los índices, los ejes del ACC y las 


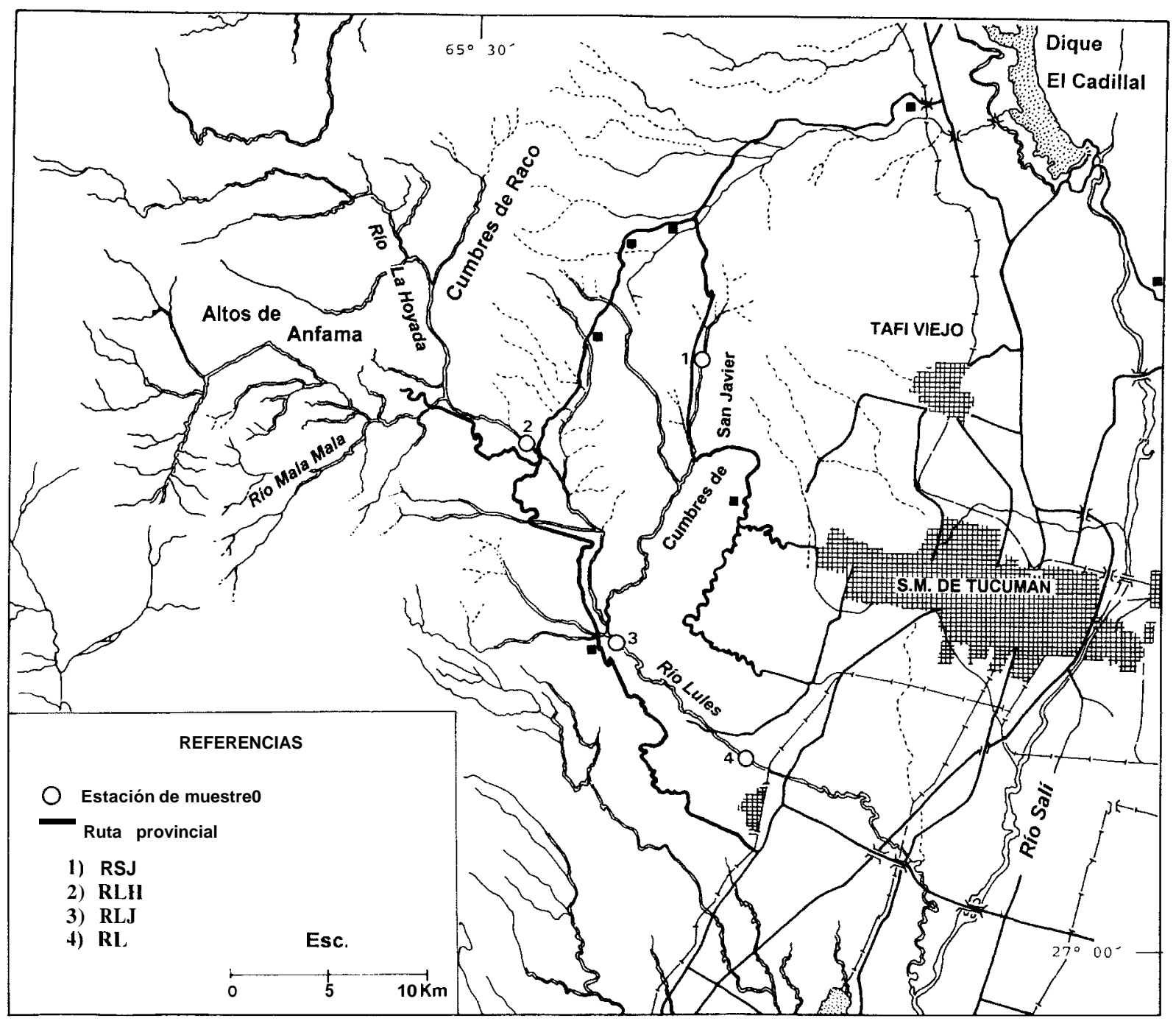

Figura 2. El Río Lules mostrando la ubicación de las cuatro estaciones de inuestreo, ríos: San Javier, La Hoyada, Las Juntas y Lules. Location of the four sampling siies in the River. Lules were San Javier stream. La Hoyada River: Las Juntas River and the Lules River itself.

variables, se utilizó el coeficiente de correlación de Spearman y su correspondiente test de significancia (Elliot, 1977).

\section{RESULTADOS}

Se identificaron 38 taxones de macroinvertebrados bentónicos de los cuales $78 \%$ pertenece a lnsecta (Tabla 2).
Los tres índices indican en general una buena calidad de agua en las cuatro estaciones (Tabla 3 ). La estación RSJ es la que muestra mayores variaciones del EPT a lo largo de los distintos muestreos. Es asimismo la única que manifiesta algún deterioro en la calidad del agua según los tres índices. La estación RLH es la que presentó mejores condiciones según los valores del EPT y el BMWP'.

En la figura 3 podemos observar como se ordenan las estaciones según los dos primeros ejes del 
Tabla 2. Matriz de presencia y ausencia de los distintos taxones en los seis muestreos realizados en cada estación. Presence / absence matrix of taxa during the six sampling conducted at each site.

\begin{tabular}{|c|c|c|c|c|c|c|c|c|c|c|c|c|c|c|c|c|c|c|c|c|c|c|c|c|}
\hline \multicolumn{25}{|c|}{ Estaciones } \\
\hline \multicolumn{7}{|c|}{ RSJ } & \multicolumn{6}{|c|}{ RLH } & \multicolumn{6}{|c|}{ RLJ } & \multicolumn{6}{|c|}{$\mathbf{R L}$} \\
\hline Taxones/Muestreos & $\mathbf{1}$ & 2 & 3 & 4 & 5 & 6 & 1 & 2 & 3 & 4 & 5 & 6 & 1 & 2 & 3 & 4 & 5 & 6 & 1 & 2 & 3 & 4 & 5 & 6 \\
\hline \multicolumn{25}{|l|}{ ODONATA } \\
\hline Zygoptera & 1 & 1 & 1 & 0 & 1 & 0 & 0 & 0 & 0 & 0 & 0 & 0 & 1 & 0 & 0 & 0 & 0 & 1 & 0 & 0 & 0 & 0 & 0 & 0 \\
\hline \multirow{2}{*}{\multicolumn{23}{|c|}{ PLECOPTERA }} & & 0 \\
\hline & & & & & & & & & & & & 1 & & & & & 0 & 1 & 1 & 1 & 0 & 1 & 1 & 1 \\
\hline \multicolumn{24}{|l|}{ EPHEMEROPTERA } & 1 \\
\hline Leptophlebiidae & 1 & 1 & 1 & 1 & 1 & 1 & 0 & 1 & 0 & 1 & 1 & 1 & 1 & 1 & 1 & 1 & 0 & 1 & 1 & 1 & 1 & 1 & 0 & 0 \\
\hline Leptohyphidae & 1 & 1 & 1 & 1 & 1 & 0 & 1 & 1 & 1 & 1 & 1 & 0 & 1 & 1 & 1 & 1 & 1 & 1 & 1 & 1 & 1 & 1 & 1 & 0 \\
\hline Caenidae & 0 & 0 & 0 & 0 & 1 & 0 & 0 & 0 & 0 & 0 & 0 & 0 & 0 & 0 & 0 & 1 & 0 & 0 & 0 & 0 & 0 & 0 & 0 & 0 \\
\hline \multicolumn{24}{|l|}{ TRICHOPTERA } & 0 \\
\hline Glossosomatidae & 0 & 1 & 0 & 1 & 0 & 0 & 1 & 1 & 1 & 1 & 1 & 1 & 1 & 1 & 1 & 0 & 1 & 0 & 0 & 1 & 0 & 0 & 0 & 0 \\
\hline Helicopsychidae & 0 & 1 & 0 & 0 & 0 & 0 & 0 & 0 & 0 & 1 & 0 & 0 & 0 & 0 & 0 & 0 & 0 & 0 & 0 & 0 & 0 & 0 & 0 & 0 \\
\hline Hydrobiosidae & o & 0 & 0 & 0 & 0 & 0 & 0 & 1 & 0 & 0 & 0 & 0 & 1 & 0 & 1 & 1 & 0 & 0 & 0 & 1 & 1 & 0 & 0 & 0 \\
\hline Hydropsychidae & 1 & 1 & 0 & 1 & 0 & 0 & 1 & 1 & 1 & 1 & 1 & 0 & 1 & 1 & 1 & 0 & 1 & 1 & 1 & 0 & 0 & 1 & 1 & 1 \\
\hline Policentropodidae & 0 & 1 & 0 & 0 & 0 & 0 & 0 & 0 & 0 & 0 & 0 & 0 & 0 & 0 & 0 & 0 & 0 & 0 & 0 & 0 & 0 & 0 & 0 & 0 \\
\hline \multicolumn{24}{|l|}{ MEGALOPTERA } & 0 \\
\hline $\begin{array}{l}\text { Corydalidae } \\
\text { COLEOPTERA }\end{array}$ & \multicolumn{18}{|c|}{ COLEOPTERA } & 1 & 1 & 0 & 0 & 0 & 0 \\
\hline Staphylinidae & 1 & 1 & 1 & 0 & 1 & 1 & 0 & 1 & 1 & 1 & 0 & 0 & 1 & 1 & 0 & 1 & 1 & 0 & 0 & 0 & 1 & 0 & 1 & 1 \\
\hline Elmidae & 1 & 1 & 0 & 1 & 0 & 1 & 1 & 1 & 1 & 1 & 1 & 1 & 1 & 1 & 1 & 1 & 1 & 1 & 1 & 1 & 1 & 1 & 1 & 1 \\
\hline Hydrophilidae & 0 & 0 & 1 & 1 & 0 & 0 & 0 & 0 & 0 & 0 & 0 & 1 & 1 & 0 & 0 & 0 & 0 & 0 & 0 & 0 & 0 & 0 & 0 & 0 \\
\hline Pcephenidae & 1 & 1 & 1 & 0 & 1 & 0 & 1 & 1 & 1 & 0 & 0 & 1 & 1 & 0 & 0 & 0 & 1 & 0 & 0 & 0 & 0 & 0 & 0 & 0 \\
\hline Dytiscidae & 0 & 0 & 0 & 0 & 1 & 0 & 0 & 0 & 0 & 0 & 0 & 0 & 0 & 0 & 0 & 0 & 0 & 0 & 0 & 0 & 0 & 0 & 0 & 0 \\
\hline Dryopidae & o & 0 & 0 & 0 & 0 & 0 & 0 & 0 & 0 & 0 & 1 & 0 & 0 & 0 & 0 & 0 & 0 & 0 & o & 0 & 0 & 0 & 0 & 1 \\
\hline \multicolumn{25}{|l|}{ DIPTERA } \\
\hline Chironomidae & 1 & 1 & 1 & 1 & 1 & 1 & 1 & 1 & 1 & 1 & 1 & 1 & 1 & 1 & 1 & 1 & 1 & 1 & 0 & 1 & 1 & 1 & 1 & 1 \\
\hline Tipulidae & 0 & 1 & 1 & 1 & 0 & 0 & 1 & 1 & 1 & 1 & 0 & 1 & 0 & 1 & 0 & 1 & 1 & 0 & 0 & 1 & 1 & 0 & 0 & 0 \\
\hline Empididae & 0 & 1 & 0 & 1 & 0 & 0 & 0 & 0 & 0 & 0 & 0 & 1 & 0 & 0 & 0 & 1 & 0 & 0 & 0 & 1 & 0 & 0 & 0 & 0 \\
\hline Ephydridae & 0 & 0 & 0 & 0 & 0 & 0 & 0 & 0 & 0 & 0 & 0 & 0 & 0 & 0 & 0 & 0 & 0 & 0 & 0 & 1 & 0 & 0 & 0 & 0 \\
\hline Simuliidae & 1 & 1 & 1 & 1 & 1 & 1 & 1 & 1 & 1 & 1 & 1 & 1 & 1 & 1 & 1 & 1 & 0 & 1 & 0 & 1 & 1 & 1 & 1 & 1 \\
\hline Psychodidae & 1 & 1 & 1 & 1 & 0 & 0 & 1 & 1 & 0 & 1 & 0 & 1 & 1 & 0 & 0 & 1 & 0 & 1 & 0 & 0 & 1 & 0 & 1 & 1 \\
\hline Ceratopogonidae & 0 & 0 & 0 & 1 & 1 & 0 & 0 & 0 & 0 & 1 & 1 & 0 & 0 & 0 & 0 & 1 & 1 & 0 & 0 & 0 & 0 & 1 & 0 & 0 \\
\hline \multicolumn{25}{|l|}{ LEPIDOPTERA } \\
\hline Pyralidae & 0 & 0 & 1 & 1 & 1 & 0 & 0 & 0 & 0 & 0 & 1 & 0 & o & 0 & 0 & 1 & 0 & 0 & 0 & 0 & 0 & 0 & 0 & 0 \\
\hline HEMIPTERA & 0 & 1 & 1 & 1 & 0 & 0 & 0 & 0 & 0 & 0 & 1 & 0 & 0 & 0 & 0 & 0 & 1 & 1 & 0 & 0 & 1 & 0 & 0 & 0 \\
\hline TURBELARIA & 0 & 1 & 1 & 1 & 0 & 0 & o & 0 & 0 & 0 & 0 & 0 & 0 & 0 & 0 & 1 & 0 & 0 & 0 & 0 & 1 & 0 & 0 & 0 \\
\hline NEMATODA & 0 & 1 & 0 & 1 & 0 & 0 & 0 & 0 & 1 & 0 & 1 & 1 & 1 & 0 & 0 & 1 & 0 & 1 & 0 & 0 & 1 & 0 & 0 & 1 \\
\hline OLIGOCHAETA & 1 & 1 & 1 & 1 & 1 & 1 & 0 & 0 & 1 & 0 & 0 & 1 & 1 & 1 & 1 & 1 & 1 & 1 & 0 & 1 & 1 & 1 & 0 & 1 \\
\hline MOLLUSCA & 0 & 0 & 0 & 1 & 0 & 0 & 0 & 0 & 0 & 0 & 0 & 0 & o & 0 & 0 & 0 & 0 & 0 & 0 & 0 & 0 & 1 & 1 & 0 \\
\hline OSTRACODA & 0 & 1 & 1 & 1 & 0 & 0 & o & 0 & 1 & 0 & 0 & 1 & 0 & 0 & 0 & 1 & 0 & 0 & 0 & 0 & 1 & 1 & 0 & 0 \\
\hline COPEPODA & 0 & 0 & 0 & 0 & 0 & 0 & 0 & 0 & 0 & 0 & 0 & 0 & o & 0 & 0 & 0 & 0 & 0 & 0 & 0 & 1 & 0 & 0 & 0 \\
\hline ACARINA & 1 & 1 & 1 & 1 & 1 & 1 & 1 & 1 & 1 & 0 & 1 & 1 & 1 & 1 & 1 & 1 & 0 & 1 & 1 & 1 & 1 & 0 & 1 & 1 \\
\hline \multicolumn{25}{|l|}{ DECAPODA } \\
\hline Aeglidae & o & 0 & 0 & 0 & 0 & 0 & o & 0 & 0 & 0 & 0 & 0 & 0 & 0 & 0 & 0 & 0 & 1 & 0 & 0 & 0 & 0 & 0 & 0 \\
\hline
\end{tabular}


Tabla 3. Valores de los distintos índices bióticos en los seis muestreos realizados en cada estación. Biotic index scores achieved at each of the six sampling stations.

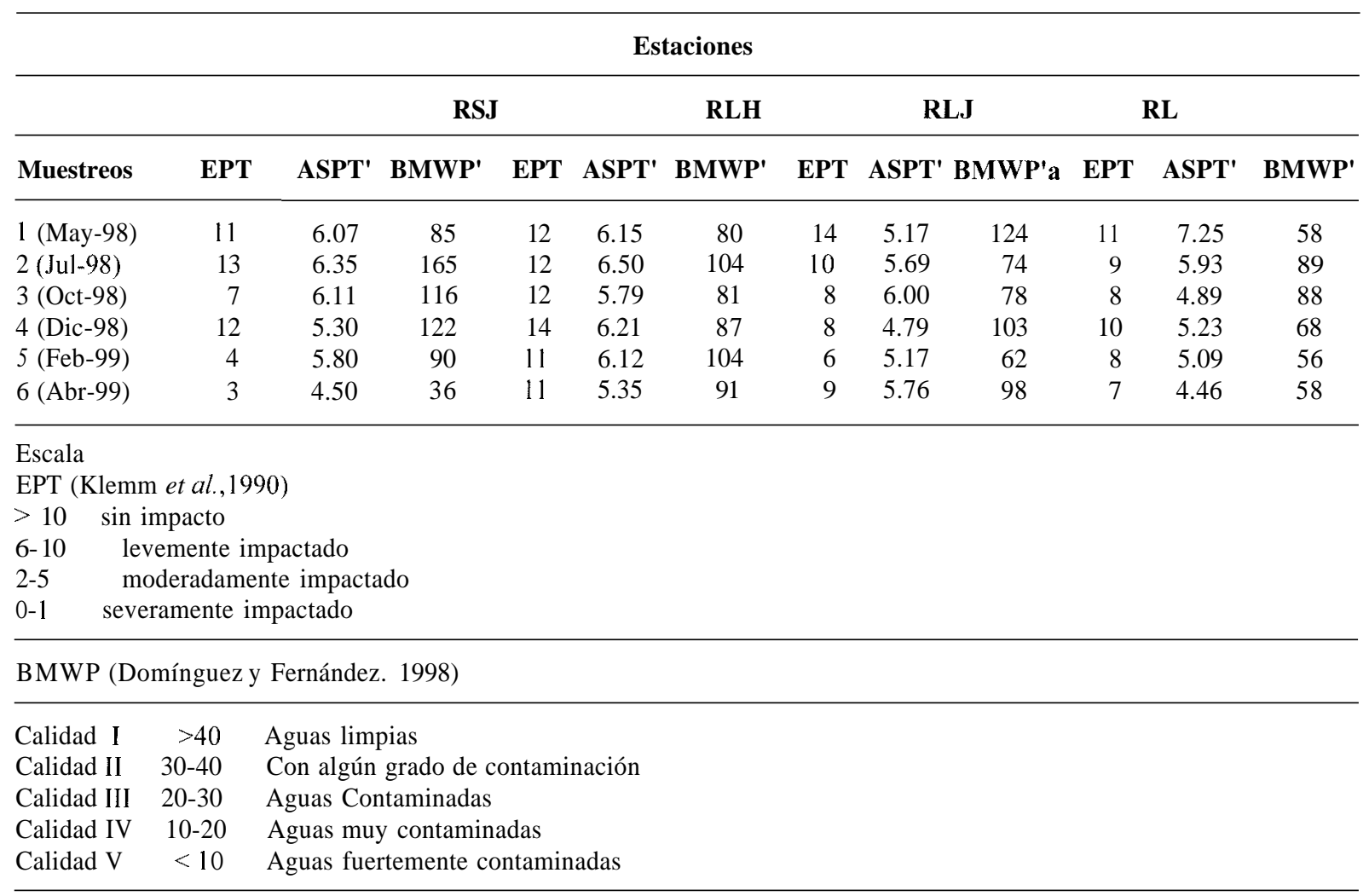

ACC. El eje I (i= 0.14) señala un gradiente de cationes $\left(\mathrm{Na}^{+}+\mathrm{K}^{+}\right)$, materia orgánica (Mat. Org.) y sólidos totales (Sól. Tot.), mientras que el eje II (1-0.11) está influenciado por el caudal.

Se puede observar que algunas estaciones (cuadrante inferior derecho) se separan al presentar una mayor concentración de NO, materia orgánica y SO,. En el cuadrante superior izquierdo se reúnen las estaciones con mejor calidad de agua.

El análisis de correlación (Tabla 4) mostró una correlación significativa $(\mathrm{p}<0.05)$ de la materia orgánica y los sólidos totales con el eje I y altamente significativa $(\mathrm{p}<0.01)$ entre el $\mathrm{Na}^{+}+\mathrm{K}^{+} \mathrm{y}$ el eje I. El eje 11 no presentó correlación significativa con ninguna de las variables aunque el caudal presenta un coeficiente alto.

$\mathrm{Al}$ considerar los índices se encuentra una correlación significativa entre el EPT y el ASPT' y altamente significativa e inversa entre el EPT y el Na'
$+\mathrm{K}^{\prime}$. El ASPT" presentó correlación significativa e inversa con el $\mathrm{Na}^{+}+\mathrm{K}^{+}, \mathrm{SO}_{4}{ }^{-}$y $\mathrm{HCO}_{3}^{-}$, mientras que el BMWP' sólo correlacionó de forma significativa e inversa con el caudal y el eje II.

En la Tabla 5 podemos observar que las aguas estudiadas presentaron valores promedios de $\mathrm{pH}$ comprendidos entre 7.58 y 8.30 , los sólidos totales oscilaron entre 191 y $429 \mathrm{mg} / \mathrm{l}$ y la materia orgánica entre 45 y $80 \mathrm{mg} / \mathrm{l}$.

El nitrato y el fosfato, presentaron valores de nitrógeno como nitrato comprendidos entre 0.02 y $1.87 \mathrm{mg} / \mathrm{l}$ y fósforo como fosfato entre 0 y 0.20 $\mathrm{mg} / \mathrm{l}$ respectivamente.

En las estaciones RSJ y RLH predomina el bicarbonato, mientras que en las estaciones RLJ y RL se increinenta notablemente el contenido de sulfato (Fig. 4).

Se observó una tendencia opuesta entre el contenido de oxígeno y los valores de materia orgá- 
Figura 3

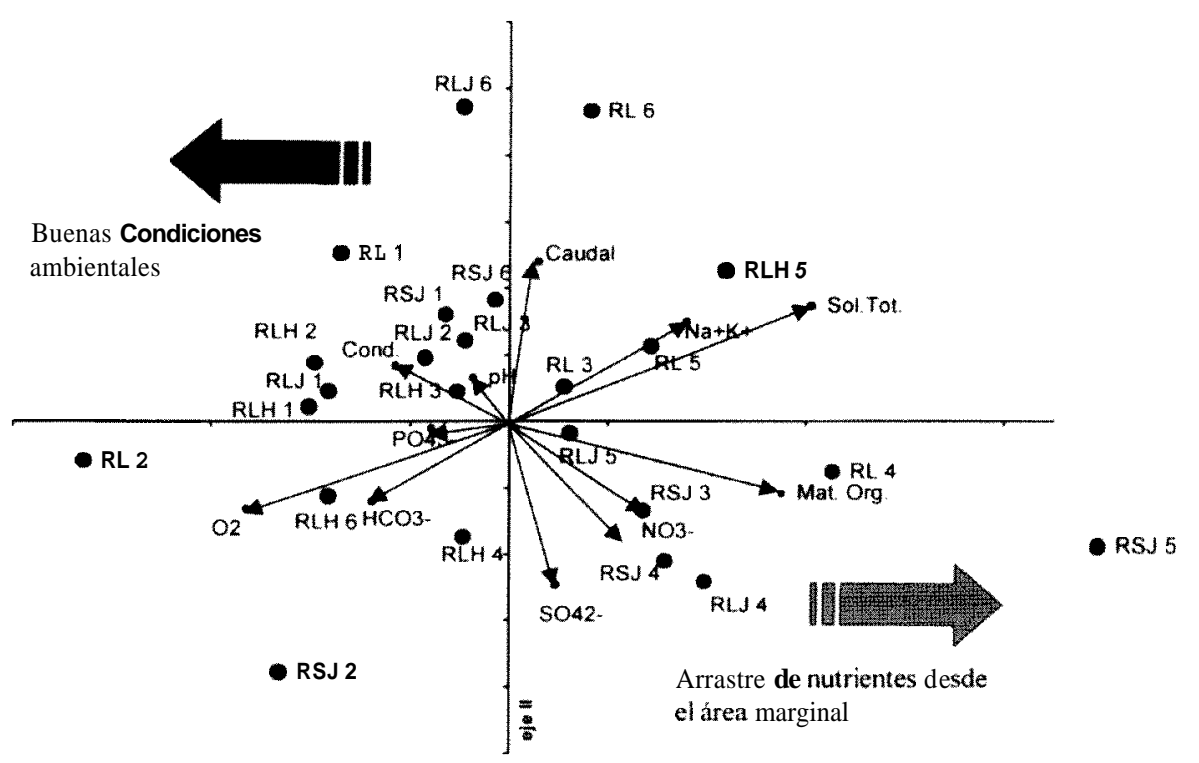

Figura 3. Diagrama de ordenación de los muestreos y las variables ambientales considerando los dos primeros ejes ( I y II ) del Análisis de Correspondencia Canónica (ACC). Ordination diagram of samplings and environmentul vuriubles on the two first axes (I \& II) of the Canonical Correspondence Analysis (CCA).

Tabla 4. Coeficientes de correlación entre las variables ambientales, los índices bióticos y los ejes I y II del Análisis de Correspondencia Canónica (ACC). Coefficient of correlation between environmentulvariables, biotic indices and the Canonical Correspondence axes, I and II.

\begin{tabular}{|c|c|c|c|c|c|}
\hline Variables & $\begin{array}{c}E j e ~ I \\
(A C C)\end{array}$ & $\begin{array}{c}\text { Eje II } \\
\text { (ACC) }\end{array}$ & EPT & ASPT ' & BMWP ' \\
\hline Oxígeno & -0.373 & -0.117 & 0.268 & 0.341 & 0.272 \\
\hline Materia Orgánica & $0.493^{*}$ & -0.181 & -0.180 & -0.008 & -0.125 \\
\hline Sólidos totales & $0.566^{*}$ & 0.210 & $-0.570^{*}$ & -0.404 & -0.201 \\
\hline Conductividad & -0.107 & 0.167 & -0.257 & -0.268 & -0.165 \\
\hline $\mathrm{Ca}^{2+}$ & -0.098 & 0.257 & -0.272 & -0.337 & -0.184 \\
\hline $\mathrm{Mg}^{2+}$ & -0.031 & -0.055 & -0.291 & -0.183 & -0.029 \\
\hline $\mathrm{Na}^{+}+\mathrm{K}^{\prime}$ & $0.643 * *$ & 0.160 & $-0.704 * *$ & $-0.483^{*}$ & -0.424 \\
\hline $\mathrm{HCO}_{3}^{-}$ & -0.146 & -0.027 & -0.050 & -0.561 * & 0.069 \\
\hline $\mathrm{Cl}^{-}$ & 0.161 & 0.177 & -0.258 & -0.334 & -0.118 \\
\hline $\mathrm{SO}_{4}^{2-}$ & 0.115 & 0.217 & -0.439 & $-0.497 *$ & -0.283 \\
\hline $\mathrm{PO}_{4}^{4-}$ & -0.154 & 0.120 & -0.094 & 0.326 & -0.034 \\
\hline $\mathrm{NO}_{3}^{4}$ & 0.085 & -0.194 & 0.018 & 0.148 & 0.080 \\
\hline $\mathrm{pH}^{3}$ & 0.021 & 0.064 & -0.263 & -0.376 & -0.213 \\
\hline caudal & -0.076 & 0.397 & 0.191 & -0.390 & $-0.499 *$ \\
\hline $\mathrm{EPT}$ & $0.538 *$ & -0.191 & & $0.524^{*}$ & 0.444 \\
\hline ASPT' & -0.446 & -0.070 & & & 0.301 \\
\hline BMWP' & -0.123 & $-0.483^{*}$ & & & \\
\hline
\end{tabular}

* $(\mathrm{p}<0.05) r t=0.48$

** $(\mathrm{p}<0.01) \mathrm{rt}=0.60$ 
Índices bióticos en Tucumán (Argentina).

Tabla 5. Parámetros fisicoquímicos de las estaciones de inuestreo a lo largo del tiempo. Temporal changes in the measured physico-chemical variables at the sampling stations

\begin{tabular}{|c|c|c|c|c|c|c|}
\hline \multicolumn{7}{|c|}{ Río San Javier (RSJ) } \\
\hline Muestreo & $\mathrm{pH}$ & $\begin{array}{l}\text { Oxígeno } \\
\left(\mathrm{mg} \mathrm{O}_{2} / \mathbf{l}\right)\end{array}$ & $\begin{array}{l}\text { Sólidos totales } \\
\text { (mg/l) }\end{array}$ & $\begin{array}{l}\text { Conductividad } \\
\qquad(\boldsymbol{\mu S} / \mathbf{c m})\end{array}$ & $\begin{array}{c}\text { Materia orgánica } \\
\text { (mg/l) }\end{array}$ & $\begin{array}{c}\text { Caudal } \\
\left(\mathrm{m}^{3} / \mathrm{s}\right)\end{array}$ \\
\hline 1 & 7.32 & 7.88 & 260 & 299 & 0 & 0.42 \\
\hline 2 & 7.96 & 8.31 & 231 & 303 & 19 & 0.46 \\
\hline 3 & 7.09 & 9.79 & 237 & 333 & 47 & 0.26 \\
\hline 4 & 7.40 & 9.15 & 215 & 283 & 24 & 0.19 \\
\hline 5 & 7.81 & 5.80 & 524 & 133 & 137 & 1.34 \\
\hline 6 & 7.88 & 7.40 & 221 & 296 & & 0.71 \\
\hline promedios & 7.58 & 8.06 & 281 & 274 & 45 & 0.56 \\
\hline \multicolumn{7}{|c|}{ Río La Hoyada (RLH) } \\
\hline Muestreo & $\mathrm{pH}$ & $\begin{array}{c}\text { Oxigeno } \\
\left(\mathrm{mg} \mathrm{O}_{\mathbf{2}} / \mathbf{l}\right)\end{array}$ & $\begin{array}{l}\text { Sólidos totales } \\
\text { (mg/l) }\end{array}$ & $\begin{array}{l}\text { Conductividad } \\
(\boldsymbol{\mu} \mathbf{S} / \mathbf{c m})\end{array}$ & $\begin{array}{l}\text { Materia orgánica } \\
\text { (mg/l) }\end{array}$ & $\begin{array}{c}\text { Caudal } \\
\left(\mathrm{m}^{3} / \mathrm{s}\right)\end{array}$ \\
\hline 1 & 7.59 & 7.51 & 124 & 148 & 44 & 4.27 \\
\hline 2 & 8.13 & 9.15 & 185 & 164 & 39 & 1.84 \\
\hline 3 & 7.47 & 8.91 & 157 & 173 & 56 & 1.73 \\
\hline 4 & 7.94 & 7.30 & 96 & 134 & 59 & 2.68 \\
\hline 5 & 7.88 & 5.80 & 455 & 114 & 175 & 8.54 \\
\hline 6 & 8.14 & 6.95 & 129 & 140 & & 6.69 \\
\hline promedios & 7.86 & 7.60 & 191 & 146 & 74 & 4.30 \\
\hline \multicolumn{7}{|c|}{ Río Las Juntas (RLJ) } \\
\hline Muestreo & $\mathrm{pH}$ & $\begin{array}{l}\text { Oxígeno } \\
\left(\operatorname{mg}_{2} / 1\right)\end{array}$ & $\begin{array}{l}\text { Sólidos totales } \\
\text { (mg/l) }\end{array}$ & $\begin{array}{l}\text { Conductividad } \\
(\boldsymbol{\mu S} / \mathbf{c m})\end{array}$ & $\begin{array}{c}\text { Materia orgánica } \\
\text { (mg/l) }\end{array}$ & $\begin{array}{c}\text { Caudal } \\
\left(\mathrm{m}^{3} / \mathrm{s}\right)\end{array}$ \\
\hline 1 & 7.60 & 8.06 & 364 & 415 & 48 & 6.69 \\
\hline 2 & 8.71 & 8.10 & 454 & 589 & 39 & 4.51 \\
\hline 3 & 7.96 & 9.52 & 387 & 591 & 73 & 3.29 \\
\hline 4 & 8.58 & 7.10 & 408 & 445 & 63 & 2.94 \\
\hline 5 & 8.03 & 5.40 & 554 & 258 & 177 & 14.50 \\
\hline 6 & 8.29 & 6.59 & 405 & 418 & & 11.55 \\
\hline promedios & 8.21 & 7.46 & 429 & 453 & 80 & 7.20 \\
\hline \multicolumn{7}{|c|}{ Río Lules (RL) } \\
\hline Mnestreo & $\mathrm{pH}$ & $\begin{array}{c}\text { Oxígeno } \\
\left(\operatorname{mg} \mathrm{O}_{2} / \mathbf{l}\right)\end{array}$ & $\begin{array}{l}\text { Sólidos totales } \\
\text { (mg/l) }\end{array}$ & $\begin{array}{l}\text { Condnctividad } \\
(\boldsymbol{\mu S} / \mathbf{c m})\end{array}$ & $\begin{array}{l}\text { Materia orgánica } \\
\text { (mg/l) }\end{array}$ & $\begin{array}{c}\text { Caudal } \\
\left(\mathrm{m}^{3} / \mathrm{s}\right)\end{array}$ \\
\hline 1 & 7.68 & 8.79 & 234 & 423 & 48 & 7.00 \\
\hline 2 & 8.66 & 8.73 & 282 & 599 & 47 & 3.24 \\
\hline 3 & 8.23 & 9.35 & 347 & 540 & 58 & 4.28 \\
\hline 4 & 8.78 & 7.10 & 409 & 448 & 71 & 6.01 \\
\hline 5 & 8.10 & 5.10 & 735 & 255 & 163 & 14.50 \\
\hline 6 & 8.35 & 6.85 & 372 & 419 & & 19.04 \\
\hline promedios & 8.30 & 7.65 & 397 & 447 & 77 & 9.00 \\
\hline
\end{tabular}




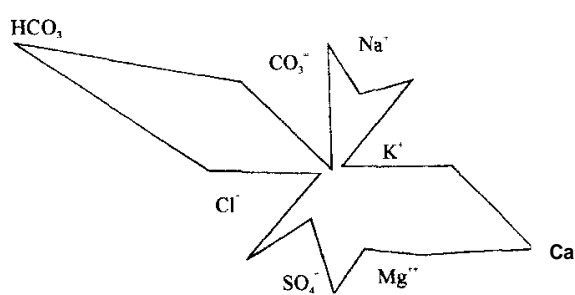

RSJ

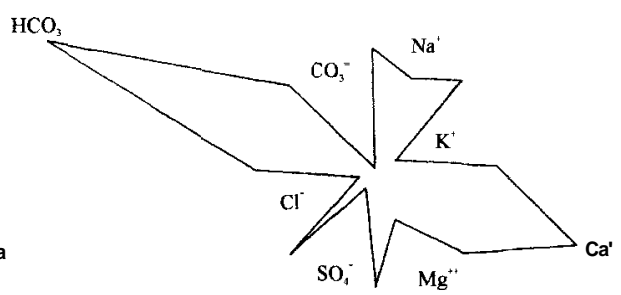

RLH
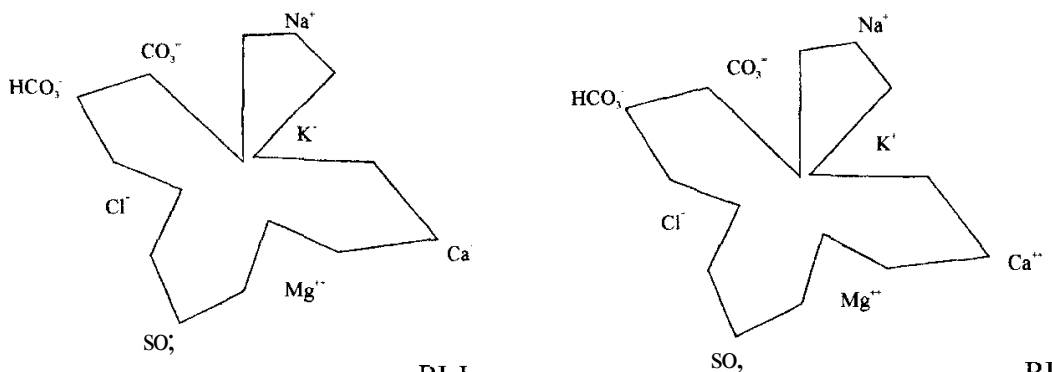

RL

Figura 4. Diagramas de Maucha correspondiente a cada una de las estaciones de muestreo. . Maucha diagrams of each sampling site.

nica así como entre aquel y el caudal a lo largo de diferentes fechas de muestreo en todas las estaciones (Fig. 5).

\section{DISCUSIÓN Y CONCLUSIONES}

La calidad del agua, medida por índices bióticos y análisis químicos, indican que no existe contaminación orgánica importante, a pesar de una primera impresión desfavorable a partir de las alteraciones visibles en las áreas marginales de por lo menos dos estaciones (RSJ y RL). Es evidente que esta situación no se manifiesta en la calidad fisico-química del agua, ni en la estructura de la comunidad bentónica.

Aunque es dificil predecir la respuesta de la comunidad (Prat y Ward, 1994), los valores de los índices (Tabla 3 ) reflejaron la alteración causada por una creciente excepcional producida por las intensas precipitaciones de mediados de marzo (1999) que causó un profundo impacto en parte de la subcuenca, haciéndose más visible en el último muestreo de las estaciones RSJ y RL donde el lecho mayor duplicó el ancho. Eventos catastróficos de esta magnitud han sido documentados en subcuencas vecinas, atribuyéndose a la intervención humana el aumento de su capacidad destructiva (Hunzinger, 1997). Si las consecuencias de este impacto se debieron a las lluvias producidas por condiciones climáticas excepcionales coincidentes con el verano-otoño de este estudio, será revelado en posteriores muestreos.

El aumento de la materia orgánica y sólidos totales en los muestreos 4 y 5 (Diciembre y Febrero) (Fig. 5) se explican por la influencia de la escorrentía de las lluvias de verano (Fig. 3) (Cooper, 1990). Es interesante destacar que dos estaciones (RSJ y RL) evidenciaron ya este impacto en el muestreo 3 (Octubre), por la influencia de las primeras lluvias de primavera. Un aumento similar pero solamente en la materia orgánica fue observado por Jacobsen (1998) en ríos de Ecuador.

Estas lluvias sin embargo no tienen el suficiente volumen para incidir significativamente en el 
RSJ

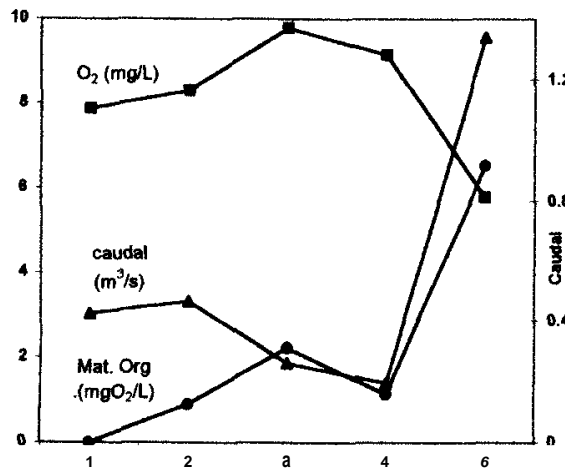

RLJ

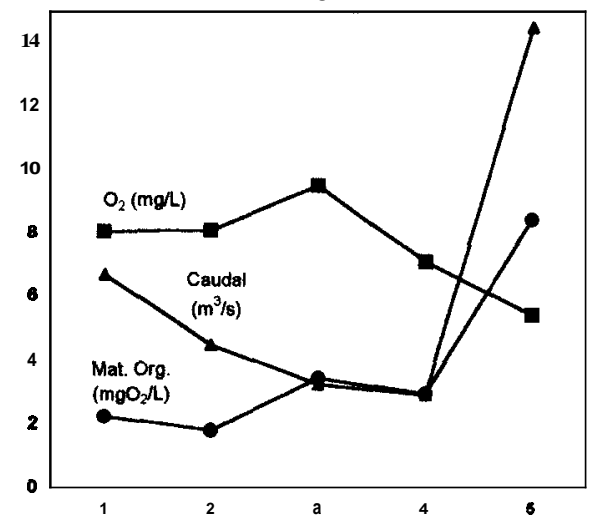

RLH

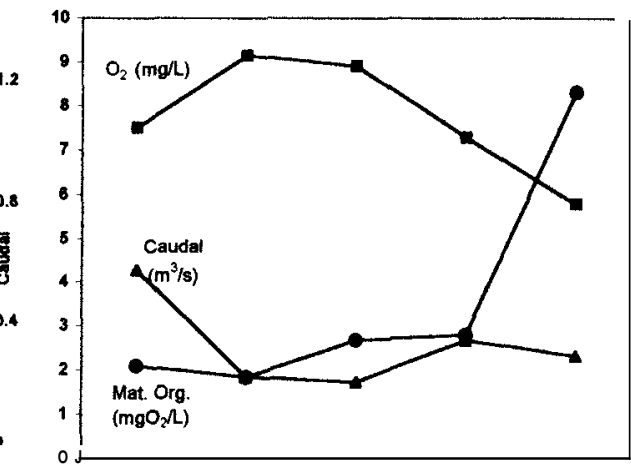

RL

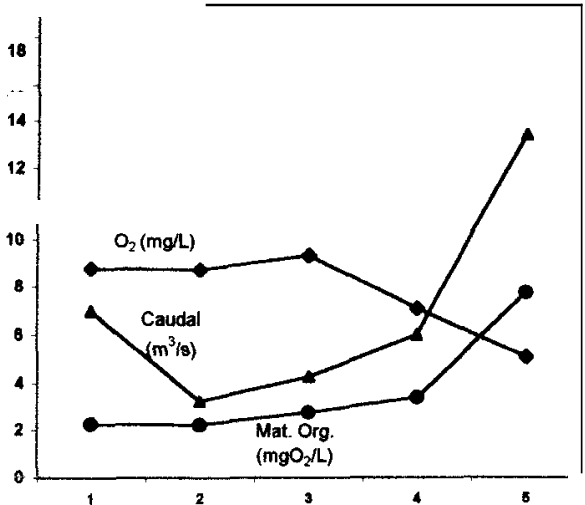

Figura 5. Variación del Oxígeno Disuelto, Materia Orgánica y Caudal en las estaciones de muestreo a lo largo del tiempo. — Oxígeno disuelto $(\mathrm{mg} / \mathrm{L})$ - Materia orgánica $(\mathrm{mg} / \mathrm{L})$ a Caudal $\left(\mathrm{m}^{3} / \mathrm{s}\right)$. Changes in Dissolved Oxigen, Organic Matter and Flow at all sampling sites . Dissolved Oxygen $\left(\mathrm{mg} \mathrm{L}^{-1}\right) \bullet$ Organic Mutter $\left(\mathrm{mg} \mathrm{L}^{-1}\right)$ A Flow $\left(\mathrm{m}^{3} \mathrm{~s}^{-1}\right)$.

caudal (Fig. 3). Esto es detectado por el EPT que se correlaciona significativamente con el eje I mostrando un importante gradiente para la materia orgánica y sólidos totales, aunque los valores de oxígeno disuelto nunca cayeron por debajo de los niveles supuestamente críticos. Este aspecto del índice es interesante porque se muestra sensible a los efectos de los sólidos totales como ya fuera observado por Salinas et al. (1999) en ríos de Yungas de Bolivia. Por otro lado es notable la correlación significativa entre este índice y el ASPT' que indicaría que ambos responden del mismo modo a las variaciones ambientales a pesar de la gran diferencia en su cálculo.

Por otro lado la disminución del caudal y una buena insolación crean aparentemente condi- ciones favorables para el crecimiento de Cladophora sp. y otras algas (Dodds \& Gudder, 1992) aumentando de este modo el proceso de fotosíntesis en el canal del río y por lo tanto el contenido de oxígeno; esto explicaría su relación inversa con el caudal (Fig. 5).

Analizando los resultados obtenidos, para los diferentes parámetros fisico-químicos y químicos determinados, observamos que los valores de: $\mathrm{pH}$, contenido de oxígeno, nutrientes analizados, se ajustan a los esperados para aguas naturales (Chapman, 1992; Allan, 1998).

Los diagramas de Maucha (Fig. 4), revelan un significativo predominio de bicarbonato en las estaciones RSJ y RLH. En las dos últimas estaciones (RLJ y RL) se incrementa notablemente el 
contenido de sulfato que es consecuencia del aporte de un afluente (río Potrero de las Tablas), cuyas aguas son sulfatadas.

Todos los parámetros analizados desde el punto de vista físico-químico conducen a valores esperados para aguas naturales en donde su naturaleza no ha sido afectada por factores exógenos de tipo antropogénicos. Sin embargo la ocurrencia de intensas precipitaciones en períodos temporales reducidos y su consiguiente influencia en los caudales de los ríos impactan en las condiciones de los hábitats de los macroinvertebrados bentónicos. En consecuencia estos reflejan alteraciones de las márgenes aunque estas no sean contaminantes.

La calidad del agua de este río, en el tramo estudiado, se puede clasificar como clase I (libre de contaminación) basándose en el índice BMWP' que, en nuestro caso, parece ser un indicador aceptable a pesar de las variaciones de caudal (Tabla 4) y los ciclos biológicos de los insectos (Zamora - Muñoz y Alba - Tercedor, 1996).

Los índices ASPT' y EPT son más sensibles que el anterior, así detectan un impacto moderado en la primera estación (RSJ) y leve en las dos últimas (RLJ y RL). El EPT además detecta los cambios de materia orgánica y sólidos totales pero requiere una mayor capacitación por parte del usuario.

\section{AGRADECIMIENTOS}

Nuestro agradecimiento a M. Peralta y C. Molineri por el apoyo en las tareas de campo y a la Dra. M.C.Apella por la lectura crítica del manuscrito.

Este trabajo se realizó con un subsidio del Consejo de Investigaciones UNT (26/G111).

\section{REFERENCIAS}

ALBA-TERCEDOR, J. y A. SÁNCHEZ-ORTEGA. 1988. Un método rápido y simple para evaluar la calidad biológica de las aguas corrientes basado en el de Hellawell (1978). Limnetica, 4: 51-56.

ALlAN, J. D. 1998. Stream Ecology Structure and Function of Running Waters. Chapman \& Hall, New York, 388 pp.
APHA. 1992. Standard Methods for the examination of water and wastewater, $18^{\text {th }}$ ed. American Public Health Association, New York.

BRAIONI, A., M.G.BRAIONI, P. DE FRANCESCHI, F. MASON, S. RUFFO, B. SAMBUCAR. 1994. Indici ambientali sintetici di valutazione della qualita delle rive (Presentazione di una scheda di relevamento). Scienza e Gobernó, 23 (1): 45-52.

CAICEDO, O. y J. PALACIO. 1998. Los macroinvertebrados bénticos y la contaminación orgánica en la quebrada de la Mosca (Guarne, Antioquia, Colombia). Actual. Biol., 20 (69): 61-73.

CAIRNS, J. \& J. R. PRATT. 1993. A history of Biological Monitoring Using Benthic Macroinvertebrates. En: Freshwater Biomonitoring and Macroinvertebrates. D. M. Rosemberg \& V. H. Resh (eds.): 10-27. Chapman \& Hall, London, 488 pp.

CHAPMAN, D. 1992. Water Quality Assessments. A guide to the biota, sediments and water in environmental monitoring. Chapman \& Hall, London, $584 \mathrm{pp}$.

COOPER, A. B. 1990. Nitrate depletion in the riparian zone and stream channei of a small headwater catchment. Hydrobiologiu, 202: 13-26.

COVICH, A. P. 1998. Geographical and historical comparisons of Neotropical streams: biotic diversity and detrital processing in highly variable habitats. J. N. Am. Benthol. Soc.,7 (4): 361-386.

DODDS, W. 1997. Distribution of runoff and river related to vegetative characteristics, latitude, and slope: a global perspective. J. N. Am . Benthol. Soc.,6(1): 162-168.

DODDS, W. \& D. A. GUDDER 1992. The ecology of Cladophora. J. Phycol.,28: 415-427.

DOMÍNGUEZ, E. y H. R. FERNÁNDEZ. 1998. Calidad de los ríos de la cuenca del Salí (TucumánArgentina) medida por un índice biótico. Serie conservación de la Naturaleza $\mathrm{n}^{\circ}$ 12. Fundación Miguel Lillo, Republica Argentina, 39 pp.

HUNZINGER, H. 1997. Hydrology of montane forest in the sierra de San Javier, Tucumán, Argentina. Mountain Research and Development, 17 (4): 299-308.

HYNES, H. B. N. 1970. The Ecology of Running Water: Liverpool University Press, $555 \mathrm{pp}$.

ELLIOT, J. M. 1977. Some methods for the Statistical Analysis of samples of Benthic Jnvertebrates. Scientific Publication of The Freshwater Biological Association, $\mathrm{N}^{\circ} 25,148 \mathrm{pp}$.

EVARSA. 1997. Estadistica hidrológica. Torno II: 509-5 12. Buenos Aires. 
GRAÇA M. A. S. \& C. N. COIMBRA. 1998. The elaboration of indices to assess biological water quality. A case study. Wat. Res., 32(2): 380-392.

JACOBSEN, D. 1998. The effect of organic pollution on the macroinvertebrate fauna of Ecuadorian highland streams. Arch. Hydrobiol., 143(2): 179-195.

KARR, J. R., L. A. TOTH \& D. R. DUDLEY. 1985. Fish communities midwestern rivers: a history of degradation. BioScience, 35: 90-95.

KLEMM, D. J., P. A. LEWIS, F. FULK \& J. M. LAZORCHAK. 1990. Macroinvertebrate field and laboratory methods for evaluating the biological integrity of surface waters. EPA/600/4-90/030. US. Environmental Protection Agency. Environmental Monitoring Systems Laboratoy, Cincinnati, Ohio 45268.

MORALES, J. M., M. SIROMBRA y A. D. BROWN. 1995. Riqueza de árboles en las Yungas argentinas. En: Investigación, Conservación y Desarrollo en Selvas Subtropicales de Montaña. A. D. Brown \& H. R. Grau (eds.): 163-174. Proyecto de Desarrollo agroforestal/ L.I.E.Y. $270 \mathrm{pp}$.

MOYANO, M. Y. y C. P. MOVIA. 1988. Relevamiento fisonómico-estructural de la vegetación de la Sierra de San Javier y el Periquillo (Tucumán, Argentina). 1: Area de las Yungas. Lilloa, 37(1): 123-135.

PRAT, N. \& J. V. WARD. 1994. The tamed river. En: Limnology now: a paradigm of planetary problems. R. Margalef (ed.): 219-236, Elsevier Science, New York.
RODIER, J. 1989. Análisis de las aguas. $6^{\text {ta }}$. Ed. Ediciones Omega, Barcelona, 1095 pp.

ROLDÁN PÉREZ, G. 1992. Fundamentos de Limnología Neotropical. Ed. Universidad de Antioquía, Colombia, 529 pp.

SALINAS, G; R. MARÍN, C. HENRY, O. FUSSATI y J. G.WASSON. 1999. Efecto de la materia en suspensión sobre los invertebrados bénticos de los ríos de aguas claras en las Yungas de Bolivia. Rev. Bol. de Ecol., 6: 5-17.

TER BRAAK, C. J. F. 1986. Canonical Correspondence Analysis: a new eigenvector technique for multivariate direct gradient analysis. Ecology, 67 (5): 1167-1179.

TER BRAAK, C. J. F. 1988. CANOCO. a FORTRAN program for canonical community ordination. Agricultural Mathematics Group. Wageningen, The Netherlands, 95 pp.

WALLEY, W. J. \& H. A. HAWKES 1997. A Computer-Based Developement of the Biological Monitoring Working Party Score System lncorporating Abundance Rating, Site Type and Indicator Value. Wat. Res. 31(2): 201-210.

ZAMORA-MUÑOZ, C. \& J. ALBA-TERCEDOR 1996. Bioassessment of organically polluted Spanish river, using biotic index and multivariated methods. J. N. Am. Benthol. Soc., 15(3): 332-352. 
